\title{
Enhanced electromagnetic pressure in a sandwiched reflection grating
}

\author{
C. P. Huang, ${ }^{1,2, *}$ S. B. Wang, ${ }^{1}$ X. G. Yin, ${ }^{3}$ Y. Zhang, ${ }^{2}$ H. Liu, ${ }^{4}$ Y. Y. Zhu, ${ }^{4}$ and C. T. Chan ${ }^{1, \dagger}$ \\ ${ }^{1}$ Department of Physics, The Hong Kong University of Science and Technology, Clear Water Bay, Kowloon, Hong Kong, China \\ ${ }^{2}$ Department of Applied Physics, Nanjing University of Technology, Nanjing 210009, China \\ ${ }^{3}$ State Key Laboratory of Millimeter Waves, City University of Hong Kong, Tat Chee Avenue, Kowloon, Hong Kong, China \\ ${ }^{4}$ National Laboratory of Solid State Microstructures, Nanjing University, Nanjing 210093, China
}

(Received 8 June 2012; published 23 August 2012)

\begin{abstract}
We suggest that strongly enhanced electromagnetic pressure can be induced at the boundary of a sandwiched reflection grating due to a special kind of deep subwavelength Fabry-Perot (FP)-like resonance. A theoretical formula will be derived and employed to study this effect at both the infrared and microwave regimes. The magnitude or sign of the electromagnetic force can be tuned by adjusting the system parameters, which in turn adjust the balance between the electric and magnetic energies stored in the system. In the infrared regime, a strong attractive pressure was found, which is controllable by the FP cavity length. In the microwave band, a repulsive pressure can be realized in most situations. What is rather amazing is that a strong attractive electromagnetic pressure, which has not been found previously, can also be demonstrated in some geometries in the microwave regime.
\end{abstract}

DOI: 10.1103/PhysRevB.86.085446

PACS number(s): 78.67.-n, 42.79.Dj, 73.20.Mf

\section{INTRODUCTION}

It is well known that charged particles can be manipulated by static electric or magnetic field gradients. With scattering or gradient forces, the optical fields of visible or infrared radiations can also exert optical forces on the small particles. ${ }^{1}$ One advantage of using optical fields is that the light intensity and thus the resulting forces can be greatly enhanced by using focused laser beams or by employing the resonances. The optical force due to the radiations may be utilized to push, trap, or even pull the small objects. ${ }^{2,3}$ In recent years there are also significant advances in the study of the optical force in the resonant cavities, where a strong optomechanical coupling between the trapped optical modes and the mechanical motion of oscillators can be established. ${ }^{4-9}$ The coupling effect may find useful applications in the amplification or cooling of the mechanical resonators.

The optical force of cavities can be boosted significantly in the plasmonic systems due to the excitation of strong plasmonic resonance modes. ${ }^{10-15}$ Interestingly, the optical fields confined by a plasmonic cavity, e.g., a simple cavity composed of two flat unstructured parallel metal plates, may serve to reduce rather than to expand the cavity size. ${ }^{13}$ Moreover, the near-field interaction between the dielectric or metal surface and a metal film, which is perforated with a periodic array of subwavelength holes, can also give rise to an attractive force. ${ }^{15}$ The unusual negative optical pressure in optical frequencies stems from the kinetic energy of free electrons, which can suppress the repulsive force due to the magnetic field in the plasmonic cavity. ${ }^{13}$ Nonetheless, the dominance of internal inductance over Faraday inductance can happen only in the high frequency regime, and as such, the electromagnetic pressure in the microwave band is usually positive because of the repulsive effect in a resonant cavity. ${ }^{14}$ The combination of electromagnetic, electronic, and mechanical degrees of freedom in the plasmonic systems may enrich the study of cavity optomechanics, leading to interesting physical properties.

Recently, we have shown that by employing the open-ended slit cavities within a simple sandwiched reflection grating, a deep subwavelength Fabry-Perot (FP)-like resonance in the metallic slits can be realized. ${ }^{16}$ The resonance wavelength can be two orders of magnitude larger than the cavity length. In this paper we suggest that such a resonance can induce an extraordinary electromagnetic pressure in the system. Theoretical formula will be derived and used to study the effect in different frequency bands. Compared with a single plasmonic cavity, ${ }^{13,14}$ more electromagnetic energy can be trapped in our periodic structure and thus may generate a stronger electromagnetic force. Moreover, our structure and results differ significantly from those of Ref. 15, where the subwavelength holes have a cutoff and the system response is dominated by the horizontal "magnetic resonance." But the metallic slits employed here have no cutoff frequency and thus a vertical FP-like resonance in the slits can be supported at very long wavelengths. Besides an efficient control of the magnitude of the electromagnetic pressure, the effect can even produce a strong negative pressure in the microwave band, depending on the amount of electric or magnetic energy stored in the slit FP cavities. The paper is organized as follows. In Sec. II the theoretical formula for the electromagnetic pressure will be deduced. In Sec. III we employ the formula to study the electromagnetic pressure in both the infrared and the microwave regimes, and the underlying mechanism will be discussed. A short summary is given in Sec. IV.

\section{THEORETICAL FORMULA}

The schematic view of the structure under study is shown in Fig. 1. Here, a dielectric-filled metallic-slit grating and a planar metal film (the thickness of metals is much larger than the skin depth) are separated by a thin dielectric spacer with a thickness of $t$. The slit grating has a periodicity of $d$, a narrow slit-width of $a$, and a thickness of $h$ (here we call $h$ the slit length). The structure is illuminated by a transverse magnetically (TM) polarized electromagnetic wave with an incident angle of $\varphi$, and the magnetic field is aligned along the $z$ axis. We assume that the permittivity of the incident side, the slit inside, the dielectric spacer, and the metal is $\varepsilon_{1}, \varepsilon_{s}, \varepsilon_{3}$, and 


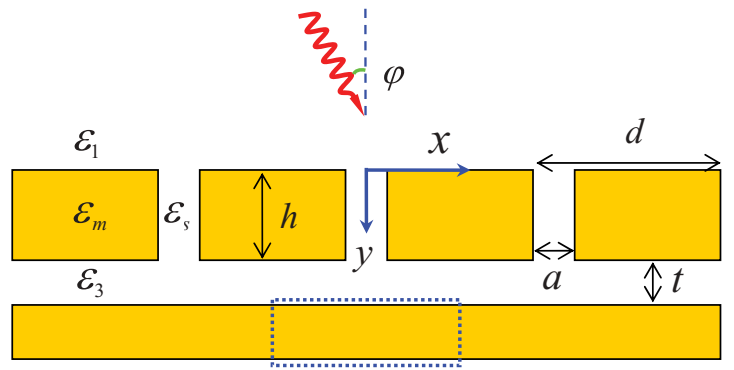

FIG. 1. (Color online) Schematic view of the sandwiched reflection grating. A thin dielectric spacer with the thickness $t$ separates a planar metal film and a metallic slit grating (the metal thickness is much larger than the skin depth). The incident light is TM-polarized with the magnetic field along the $z$ axis. The dashed line represents a cubic box for calculating the electromagnetic pressure.

$\varepsilon_{M}$, respectively. Note that similar structures have been studied previously in the regime $h \ll t,{ }^{17-20}$ where the spacer region below the metal stripes is dominant and a series of horizontal FP-like resonances in the spacer can be supported. In the following we focus on the opposite regime $h \gg t$ and $\lambda \gg h$, where deep subwavelength FP-like resonances in the metallic slits can be established. ${ }^{16}$ To deduce the electromagnetic pressure, we employ the Maxwell-stress-tensor method, which requires a determination of electromagnetic fields in the spacer.

\section{A. Field expansions}

\section{Incident side}

The incident electromagnetic wave will be scattered by the periodic interface and the electromagnetic fields on the incident side, including the incoming light and scattering components, can be expressed with the Fourier expansion as follows:

$$
\begin{aligned}
& H_{3}^{\text {in }}=e^{i k_{0}\left(\gamma_{0} x+u_{0} y\right)}+\sum_{m} R_{m} e^{i k_{0}\left(\gamma_{m} x-u_{m} y\right)}, \\
& E_{1}^{\text {in }}=\frac{1}{\varepsilon_{0} \varepsilon_{1} c}\left(-u_{0} e^{i k_{0}\left(\gamma_{0} x+u_{0} y\right)}+\sum_{m} R_{m} u_{m} e^{i k_{0}\left(\gamma_{m} x-u_{m} y\right)}\right) .
\end{aligned}
$$

Here and in the following, the subscripts 1, 2, and 3 in the electromagnetic fields indicates $x, y$, and $z$ components, respectively; $R_{m}$ is the amplitude of the $m$ th-order diffraction mode on the incident side; $\gamma_{m}=\sqrt{\varepsilon_{1}} \sin \varphi+m \lambda / d, u_{m}=$ $\sqrt{\varepsilon_{1}-\gamma_{m}^{2}}$, and $k_{0}=\omega / c$ is the wavevector in free space.

\section{Metallic slits}

We are interested in those narrow slits that satisfy the conditions $a \ll \lambda$ and $h \geqslant a$. In this case, the high-order waveguide modes are rapidly decaying and can be neglected. ${ }^{21}$ Thus we only consider the fundamental slit mode:

$$
\begin{aligned}
H_{3}^{s l} & =\left(A e^{i q_{0} y}+B e^{-i q_{0} y}\right) \cos \beta x, \\
E_{1}^{s l} & =\frac{q_{0}}{\varepsilon_{0} \varepsilon_{s} \omega}\left(-A e^{i q_{0} y}+B e^{-i q_{0} y}\right) \cos \beta x, \\
E_{2}^{s l} & =\frac{i \beta}{\varepsilon_{0} \varepsilon_{s} \omega}\left(A e^{i q_{0} y}+B e^{-i q_{0} y}\right) \sin \beta x .
\end{aligned}
$$

Here, $A$ and $B$ are the amplitudes of the downward and upward waves in the slits, $q_{0}=\sqrt{k_{0}^{2} \varepsilon_{s}-\beta^{2}}$ is the longitudinal propagation constant of the slit mode, and $\beta$ is the transverse eigenwave vector, which will be determined below. The single mode approximation can also be justified in Sec. III, where an excellent agreement between the analytical treatment and rigorous simulation is achieved.

In the infrared and low-frequency band, the surface of a metal film (with the thickness much larger than the skin depth) can be treated by using the surface- impedance boundary condition (SIBC) $\vec{E}_{t}=Z \vec{n} \times \vec{H}_{t} \cdot{ }^{22}$ Here $\vec{E}_{t}$ and $\vec{H}_{t}$ are the tangential components of electromagnetic fields, $Z=\mu_{0} c / \sqrt{\varepsilon_{M}}$ is the surface impedance of metals, and $\vec{n}$ denotes the unit vector along the outgoing normal of the metal surface. By using Eq. (2) and imposing the SIBC on the slit walls $(x= \pm a / 2)$, we obtain $\tan (\beta a / 2)=k_{0} \varepsilon_{s} / i \beta \sqrt{\varepsilon_{M}}$. For the narrow slits, this equation leads to $\beta \approx \sqrt{2 k_{0} \varepsilon_{s} / i a \varepsilon_{M}^{1 / 2}}$. Because of the negative value of metal permittivity in the optical regime (neglecting the absorption of metal), $\beta$ will become a purely imaginary number (the wave is evanescent in the $x$ direction due to the coupling between the photons and surface charges). This suggests the fundamental slit mode is a surface-plasmon polariton (SPP) wave bounded to the slit walls. The propagation constant can thus be deduced as $q_{0}=k_{0} n_{h}$, where $n_{h}=\sqrt{\varepsilon_{s}\left(1+2 i / k_{0} a \varepsilon_{M}^{1 / 2}\right)}$ is the effective index of slits. In the near- and mid-infrared frequency range where $\varepsilon_{M} \approx-\omega_{p}^{2} / \omega^{2}$, the effective index can be reduced to $n_{h}=\sqrt{\varepsilon_{s}(1+2 \delta / a)}$, where $\delta=c / \omega_{p}$ is the skin depth of metal. This agrees with the result obtained by Collin et al. ${ }^{23}$ Also, at the microwave frequencies where $\varepsilon_{M} \approx i \sigma / \varepsilon_{0} \omega$, the effective index becomes $n_{h} \approx \sqrt{\varepsilon_{s}}(1+\delta / 2 a)$ (here the imaginary part has been neglected), where $\delta=\sqrt{2 / \mu_{0} \omega \sigma}$ is the skin depth in the microwave band. Generally, the plasmonic regime is considered to be at the visible and the near-infrared band. However, the modified index, as mentioned previously, suggests that the field penetration effect has physical consequences in the mid-infrared or even microwave region. ${ }^{23,24}$

\section{Spacer region}

The electromagnetic fields entering the spacer region will not only be scattered by the periodic structure but will also be reflected by the bottom planar metal film. The fields are thus expanded as follows:

$$
\begin{aligned}
& H_{3}^{s p}=\sum_{m} T_{m}\left(e^{i k_{0} v_{m}(y-h)}-\rho_{m} e^{-i k_{0} v_{m}(y-h-2 t)}\right) e^{i k_{0} \gamma_{m} x}, \\
& E_{1}^{s p}=\frac{-1}{\varepsilon_{0} \varepsilon_{3} c} \sum_{m} v_{m} T_{m}\left(e^{i k_{0} v_{m}(y-h)}+\rho_{m} e^{-i k_{0} v_{m}(y-h-2 t)}\right) e^{i k_{0} \gamma_{m} x}, \\
& E_{2}^{s p}=\frac{1}{\varepsilon_{0} \varepsilon_{3} c} \sum_{m} \gamma_{m} T_{m}\left(e^{i k_{0} v_{m}(y-h)}-\rho_{m} e^{-i k_{0} v_{m}(y-h-2 t)}\right) e^{i k_{0} \gamma_{m} x} .
\end{aligned}
$$

Here, $T_{m}$ is the amplitude of the $m$ th-order diffraction mode in the spacer, $v_{m}=\sqrt{\varepsilon_{3}-\gamma_{m}^{2}}$, and $\rho_{m}$ is the unknown modereflection coefficient at the surface of planar metal film.

By using Eq. (3) and applying the SIBC on the planar metal surface $(y=h+t)$, the mode-reflection coefficient can 
be determined as

$$
\rho_{m}=\left(1-\tau_{m}\right) /\left(1+\tau_{m}\right),
$$

where $\tau_{m}=v_{m} \sqrt{\varepsilon_{M}} / \varepsilon_{3}$. Here we recall the Fresnel formula, which shows that the field reflection coefficient at the semi-infinite dielectric/metal $\left(\varepsilon_{3} / \varepsilon_{M}\right)$ interface reads $\rho=$ $\left(\sqrt{\varepsilon_{3}} \cos \theta_{r}-\sqrt{\varepsilon_{M}} \cos \theta_{i}\right) /\left(\sqrt{\varepsilon_{3}} \cos \theta_{r}+\sqrt{\varepsilon_{M}} \cos \theta_{i}\right)$. Such a reflection coefficient $|\rho|$ is usually equal to or less than unity. If we have an imaginary value of $\cos \theta_{i},|\rho|$ could be larger than unity (note that $\varepsilon_{M}$ is negative and large, and, according to the Snell's law, the refraction angle $\theta_{r}$ is close to zero). In the "periodic" spacer region, one can write down $\cos \theta_{i}=v_{m} / \sqrt{\varepsilon_{3}}$. Accordingly, Eq. (4) can be recovered by using the Fresnel formula. Moreover, when the $m$ th-order diffraction mode is evanescent, $\cos \theta_{i}$ becomes imaginary (and $\tau_{m}$ is negative). This means, due to the excitation of surface charges, an evanescent incident wave may be enhanced when "reflecting" from a metal surface.

\section{B. Enhanced fields due to the FP-like effect}

The electromagnetic fields in the space can be determined by using four boundary conditions. ${ }^{25,26}$ The first two conditions lie in the continuous of magnetic field $\mathrm{H}_{3}$ at the upper and lower slit openings $(y=0, h ;-a / 2 \leqslant x \leqslant a / 2)$. By using Eqs. (1)-(3), one obtains

$$
\begin{gathered}
A+B=g_{0}+\sum_{m} g_{m} R_{m}, \\
A e^{i q_{0} h}+B e^{-i q_{0} h}=\sum_{m}\left(1-\rho_{m} e^{2 i k_{0} v_{m} t}\right) g_{m} T_{m},
\end{gathered}
$$

where $g_{m}=\sin c\left(k_{0} \gamma_{m} a / 2\right)$. The second two conditions are continuous of $\left(E_{1}+Z H_{3}\right)_{y=0}$ and $\left(E_{1}-Z H_{3}\right)_{y=h}$ at the upper and lower zone boundaries $(y=0, h ;-d / 2 \leqslant x \leqslant d / 2)$. Note that these two boundary conditions result from a matching of electromagnetic fields at the slit openings and the SIBCs at the horizontal metal surfaces of the slit grating (in the calculation, $E_{1} \pm \mathrm{ZH}_{3}$ is set as zero inside the metal surface, according to the SIBC). By further utilizing Eqs. (1)-(3) and the orthogonal condition $(1 / d) \int_{-d / 2}^{d / 2} e^{i k_{0}\left(\gamma_{m}-\gamma_{n}\right) x} d x=\delta_{m n}$, we have

$$
\begin{aligned}
R_{m} & =\kappa \delta_{m 0}-\frac{\alpha g_{m}}{1+\sigma_{m}}(A-B), \\
T_{m} & =\frac{\alpha g_{m}\left(A e^{i q_{0} h}-B e^{-i q_{0} h}\right)}{\left(1+\tau_{m}\right)\left(1-\rho_{m}^{2} e^{2 i k_{0} v_{m} t}\right)} .
\end{aligned}
$$

Here, $\sigma_{m}=u_{m} \sqrt{\varepsilon_{M}} / \varepsilon_{1}, \quad \kappa=\left(\sigma_{0}-1\right) /\left(\sigma_{0}+1\right)$, and $\alpha=$ $\left(a n_{h} / d \varepsilon_{s}\right) \sqrt{\varepsilon_{M}}$. By combining Eqs. (5) and (6) the fields in the space can thus be determined.

One of the most important quantities is the zero-order reflection $r_{0}=\left|R_{0}\right|^{2}$, which can be derived as follows: ${ }^{16}$

$$
r_{0}=\left|\kappa-\frac{\eta\left(1+r_{3} e^{2 i q_{0} h}\right)}{\left(1+\theta_{1}\right)\left(1-r_{1} r_{3} e^{2 i q_{0} h}\right)}\right|^{2} .
$$

Here, $\eta=2 g_{0}^{2} \alpha \sigma_{0} /\left(1+\sigma_{0}\right)^{2}$, and $r_{j=1,3}=\left(1-\theta_{j}\right) /\left(1+\theta_{j}\right)$ is the equivalent reflection coefficient of the slit mode at the slit openings (the slit outside behaves like an effective medium with a relative impedance of $\left.1 / \theta_{j}\right)$, where $\theta_{1}=$ $\alpha \sum_{m} g_{m}^{2} /\left(1+\sigma_{m}\right), \theta_{3}=\alpha \sum_{m} \mu_{m} g_{m}^{2} /\left(1+\tau_{m}\right)$, and $\mu_{m}=$ $\left(1-\rho_{m} e^{2 i k_{0} v_{m} t}\right) /\left(1-\rho_{m}^{2} e^{2 i k_{0} v_{m} t}\right)$.
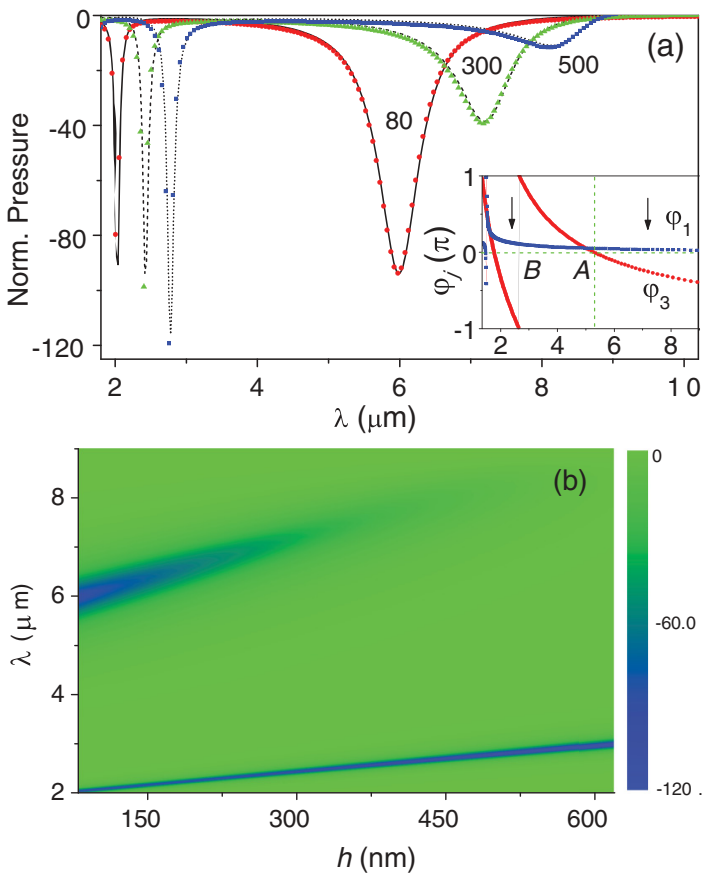

FIG. 2. (Color online) (a) Normalized electromagnetic pressure in the infrared regime for different slit length $(h=80,300$, and $500 \mathrm{~nm}$ ). The lines and symbols represent the analytical and numerical results, respectively. The inset shows the dependence of the phase shift $\varphi_{j}$ on the wavelength (the arrows indicate the positions of deep subwavelength FP-like resonances for $h=300 \mathrm{~nm}$ ). (b) Normalized electromagnetic pressure in the infrared regime as a function of wavelength and slit length. Here, the structure parameters are fixed as $d=1500 \mathrm{~nm}, a=50 \mathrm{~nm}$, and $t=20 \mathrm{~nm}$.

The reflection of wave is related to an FP-like resonance factor $f_{p}=\left(1-r_{1} r_{3} e^{2 i q_{0} h}\right)^{-1}$, suggesting that the open-ended metallic slits may act as resonant cavities when the condition $\varphi_{1}+\varphi_{3}+2 q_{0} h=2 \pi m$ is satisfied (here $\varphi_{1}$ and $\varphi_{3}$ represent the phase shift of the slit mode at the upper and lower slit openings, respectively). We have shown previously that, by using the unusual phase shifts $\varphi_{1}$ and $\varphi_{3}$, a deep subwavelength, typically zero-order FP-like resonance in the slits may be realized. ${ }^{16}$ The inset of Fig. 2 gives an example of the phase shift for this scenario. There, the phase jump near point B, corresponding to the poles of $\mu_{m}$ and $\theta_{3}$, is due to the extended SPP mode on the planar metal film. ${ }^{27}$ And, the zero-phase point A, corresponding to a near-zero $\theta_{3}\left[\operatorname{Im}\left(\theta_{3}\right)=0\right]$, is correlated with the horizontal FP-like resonance beneath the metal stripes [the spectral positions can be approximated by $2 n_{t}(d-a)=$ $m \lambda$, where $n_{t}=\sqrt{\varepsilon_{3}\left(1+2 i / k_{0} t \varepsilon_{M}^{1 / 2}\right)}$ is the effective index of the metal-dielectric-metal region]. The wavelength of the slit FP-like resonance is strongly dependent on, but can be two orders of magnitude larger than, the cavity length. ${ }^{16}$ At the resonance, the reflection of wave will be strongly suppressed, and a significant portion of energy can be stored in the slit cavity.

Another most important quantity is the amplitude of the $m$ th-order diffraction mode in the dielectric spacer, which can be expressed as

$$
T_{m}=\frac{g_{m}\left[\left(1+\theta_{1}\right)\left(1+\theta_{3}\right)\right]^{-1}}{\left(1+\tau_{m}\right)\left(1-\rho_{m}^{2} e^{2 i k_{0} v_{m} t}\right)} \chi f_{p} e^{i q_{0} h},
$$


where $\chi=4 \alpha g_{0} /\left(1+\sigma_{0}^{-1}\right)$. Equation (8) suggests that the electromagnetic fields in the dielectric spacer are also proportional to the FP-like resonance factor $f_{p}$. This demonstrates, due to the coupling effect, that the fields in the spacer will be enhanced at the vertical slit FP-like resonance but not at their inherent horizontal resonance. This is not difficult to understand. The entrance channels of the electromagnetic wave are controlled now by the vertical FP effect, and the nonresonant FP cavities will block the incident photons. Only near the vertical FP-like resonance can the electromagnetic wave be "grasped" efficiently by the subwavelength slits and then compressed into the dielectric spacer. In the following, Eq. (8) will be employed for the calculation of electromagnetic pressure.

\section{Electromagnetic pressure}

The deep subwavelength FP-like resonance and the strong field-enhancement in the metallic slit cavities as well as in the spacer region may induce an enhanced electromagnetic pressure on the planar metal film. This enhanced pressure can transfer the energy efficiently from the electromagnetic motion to the mechanical counterpart. For simplicity, here we assume that the dielectric of the spacer region is air with a permittivity of unity $\left(\varepsilon_{3}=1\right)$. To derive the electromagnetic pressure analytically, we choose a cubic box just enclosing the planar metal film (see the dash line in Fig. 1) and integrate the Maxwell stress tensor over the whole box. ${ }^{28}$ As the electromagnetic fields inside/below the metal film are very weak and can be neglected, the surface integral only needs to run over the top surface of the cubic box. Thus the $y$ component of electromagnetic force exerted on the planar metal film becomes $F_{2}=-\int T_{22} d s$, where $T_{22}=\varepsilon_{0}\left(E_{2}^{2}-E^{2} / 2\right)+\mu_{0}\left(H_{2}^{2}-H^{2} / 2\right)$ is the element of the Maxwell stress tensor. By further neglecting the $x$ component of electric field (which is very small near the metal surface) and making a time-average over an oscillating period, the time-averaged electromagnetic pressure can be written as

$$
P_{a}=(4 d)^{-1} \int_{-d / 2}^{d / 2}\left(-\varepsilon_{0}\left|E_{2}\right|^{2}+\mu_{0}\left|H_{3}\right|^{2}\right) d x,
$$

where $E_{2}$ and $H_{3}$ are the electromagnetic fields in the spacer (near the planar metal surface). One can see that the electromagnetic pressure in this structure is related to the electromagnetic energy densities, where the electric part contributes a negative (attractive) force and the magnetic part a positive (repulsive) force. The difference between the average electric and magnetic energy in the spacer has two origins: one is the kinetic energy shared by the free electrons due to the plasmonic resonance, and the other is a "leakage" of electric or magnetic energy from the spacer to the surrounding environment, especially the metallic slits acting as the FP cavities.

By using Eq. (3), the space average of $\left|E_{2}\right|^{2}$ near the metal surface $(y \sim h+t)$ can be determined in the following way:

$$
\begin{aligned}
\left|E_{2}\right|_{\mathrm{av}}^{2}= & (1 / d) \int_{-d / 2}^{d / 2} E_{2} E_{2}^{*} d x \\
= & \frac{1}{\varepsilon_{0}^{2} c^{2} d} \int_{-d / 2}^{d / 2} \sum_{m} \gamma_{m}\left[T_{m}\left(1-\rho_{m}\right) e^{i k_{0} v_{m} t}\right] \\
& \times e^{i k_{0} \gamma_{m} x} \sum_{n} \gamma_{n}\left[T_{n}\left(1-\rho_{n}\right) e^{i k_{0} v_{n} t}\right]^{*} e^{-i k_{0} \gamma_{n} x} d x
\end{aligned}
$$

$$
\begin{aligned}
= & \frac{1}{\varepsilon_{0}^{2} c^{2} d} \int_{-d / 2}^{d / 2} \sum_{m n} \gamma_{m} \gamma_{n}\left[T_{m}\left(1-\rho_{m}\right) e^{i k_{0} v_{m} t}\right] \\
& \times\left[T_{n}\left(1-\rho_{n}\right) e^{i k_{0} v_{n} t}\right]^{*} e^{i k_{0}\left(\gamma_{m}-\gamma_{n}\right) x} d x \\
= & \frac{1}{\varepsilon_{0}^{2} c^{2}} \sum_{m} \gamma_{m}^{2}\left|T_{m}\left(1-\rho_{m}\right) e^{i k_{0} v_{m} t}\right|^{2} .
\end{aligned}
$$

Here the superscript $*$ denotes the complex conjugation. In the calculation the mode orthogonal condition $(1 / d) \int_{-d / 2}^{d / 2} e^{i k_{0}\left(\gamma_{m}-\gamma_{n}\right) x} d x=\delta_{m n}$ has been used. Similarly, the space average of $\left|H_{3}\right|^{2}$ can also be calculated as

$$
\left|H_{3}\right|_{\mathrm{av}}^{2}=\sum_{m}\left|T_{m}\left(1-\rho_{m}\right) e^{i k_{0} v_{m} t}\right|^{2} .
$$

To show the order of magnitude or the efficiency of electromagnetic pressure enhancement, we normalize the electromagnetic pressure in our system to the pressure acting on an unstructured perfect metal surface by the same source. By substituting Eqs. (10) and (11) into Eq. (9), the normalized electromagnetic pressure can thus be deduced as

$$
\frac{P_{a}}{P_{0}}=\frac{1}{4} \sum_{m}\left(1-\gamma_{m}^{2}\right)\left|T_{m}\left(1-\rho_{m}\right) e^{i k_{0} v_{m} t}\right|^{2},
$$

where $P_{0}=\mu_{0}$ is the common electromagnetic pressure of a normally incident wave exerted on the flat surface of a perfect electric conductor (the incident magnetic field $\mathrm{H}_{3}$ is set as unity). Equation (12) suggests that each diffraction component in the air spacer will give a contribution to the electromagnetic pressure. As can be seen from Eq. (8), the amplitude of the diffraction mode in the spacer, $T_{m}$, is proportional to the FPlike resonance factor $f_{p}$. Thus, the electromagnetic pressure will be greatly enhanced at the reflection minimum or the deep subwavelength FP-like resonance.

\section{RESULTS AND DISCUSSIONS}

\section{A. Electromagnetic pressure in the infrared regime}

In the infrared regime the structure parameters are fixed as $d=1500 \mathrm{~nm}$ and $a=50 \mathrm{~nm}$. The relative permittivity of the incident side, the slit inside, and the spacer region were assumed to be unity, and the metal (gold) was modeled with a Drude dispersion $\varepsilon_{m}=1-\omega_{p}^{2} / \omega(\omega+i \gamma)$, where $\omega_{p}=1.37 \times 10^{16} \mathrm{rad} / \mathrm{s}$ and $\gamma=5 \times 10^{13} \mathrm{rad} / \mathrm{s}^{29}$ In the following we will calculate the electromagnetic pressure by using Eqs. (8) and (12). And, as a comparison, we also simulated the electromagnetic pressure employing the commercial software package COMSOL Multiphysics. Figure 2(a) shows the analytically calculated (lines) and numerically simulated (symbols) normalized electromagnetic pressure for different slit lengths $h=80,300$, and $500 \mathrm{~nm}$ (here the spacer thickness is set as $t=20 \mathrm{~nm}$ ). One can see that an excellent agreement between them can be achieved. Thus, our theoretical formula may provide an accurate and very efficient tool for exploring the electromagnetic force in the structure.

Figure 2(a) shows that strong and negative electromagnetic pressure can be induced at the (deep) subwavelength FP-like resonances. For the slit-cavity length $h=80 \mathrm{~nm}$, for example, the normalized electromagnetic pressure reaches -90 and -94 at the two resonance wavelengths around 2000 and $6000 \mathrm{~nm}$, 

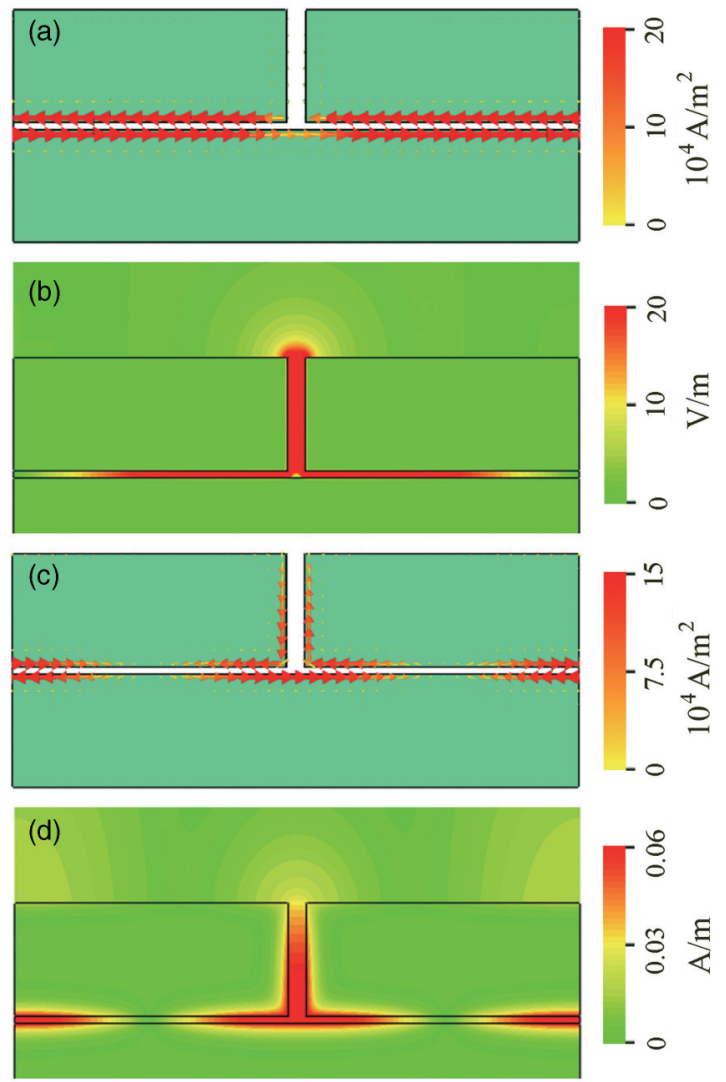

FIG. 3. (Color online) (a) Current and (b) electric field $|E|$ distribution for the deep subwavelength FP-like resonance at $7180 \mathrm{~nm}$; (c) current and (d) magnetic-field $|H|$ distribution for the FP-like resonance at $2400 \mathrm{~nm}$. Here, $h=300 \mathrm{~nm}$ and $t=20 \mathrm{~nm}$.

respectively. As we are normalizing the electromagnetic pressure to that of a flat metal surface, the value is nearly two orders of magnitude larger than that achieved on an unstructured metal surface. With the increase of $h$, the spectral positions of the two resonances shift significantly to the longer wavelength. ${ }^{16}$ Meanwhile, the electromagnetic pressure changes accordingly: the pressure at the long wavelength is reduced and that at the short wavelength increases instead. This character can also be seen clearly from Fig. 2(b), where the normalized electromagnetic pressure is determined analytically and mapped as a function of wavelength and slit length. The dark or blue-colored part in the figure highlights the strong and negative electromagnetic pressure induced by the subwavelength FP-like resonances.

To understand the previous effects, we have plotted the current density and electromagnetic field distributions for the subwavelength FP-like resonances. Taking $h=300 \mathrm{~nm}$ as an example, Fig. 3 presents the simulation results obtained by using the commercial software CST Microwave Studios. One can see from both Figs. 3(a) $(\lambda=7180 \mathrm{~nm})$ and 3(c) $(\lambda=2400 \mathrm{~nm})$ that antiparallel currents (along the $x$ direction) are excited above and below the air spacer, which induce a repulsive (positive) force on the planar metal film. Simultaneously, the positive and negative charges accumulate near the air spacer, thus giving rise to an attractive or negative force. This competition of positive and negative force can also be seen from Eq. (9), where the electric-field energy is related to the attractive force and the magnetic-field energy to the repulsive force. If there is no electric field penetration (the kinetic energy of electrons is zero) and if edge effects can be ignored, the attractive force due to electric field and the repulsive force due to magnetic field essentially cancel each other. However, field penetration in the plasmonic regime suppresses the magnetic field, as the inductance is dominated by the kinetic inductance rather than Faraday inductance. ${ }^{13}$ Such an effect may hold from the visible to mid-infrared frequencies. ${ }^{30}$ Therefore, a lot of energy is stored as the kinetic energy of free electrons, and thus the magnetic energy is less than the electric one. Consequently, a net negative force can be observed here.

To explain the different evolution behaviors of electromagnetic force at the short and long resonance wavelengths, we resort to the current and field distributions around the subwavelength slits. For the long-wavelength excitation, the current near the metal slit is very weak [see Fig. 3(a)], and a lot of electric field energy can be stored in the slits because of the deep subwavelength FP-like resonance [Fig. 3(b)]. However, for the short-wavelength resonance, the current near the metal slit becomes relatively strong due to larger phase retardation in the slit [Fig. 3(c)]; correspondingly, a lot of magnetic field energy is stored in the slits [Fig. 3(d)]. This suggests that the subwavelength slits may act as a capacitor of electric or magnetic field energy, and that the "leakage" of electric or magnetic energy from the spacer to the slits can be employed to modulate the electromagnetic force efficiently. The leakage of electric field energy into the slit can reduce the attractive force. Thus with the increase of slit length, more and more electric field energy is localized in the slit, and the net electromagnetic force is decreased (see Fig. 2). On the contrary, the leakage of magnetic field energy into the slit can reduce the repulsive force. Consequently, with the increase of slit length, more and more magnetic field energy is confined in the slit, and the total electromagnetic force will be increased instead.

Figure 4(a) presents the normalized electromagnetic pressure as a function of wavelength and spacer thickness (the slit length is set as $h=80 \mathrm{~nm}$ ) in the infrared regime. With the decrease of spacer thickness, the zero-phase point $A$ [refer to inset of Fig. 2(a)] will shift to a longer wavelength. Correspondingly, the associated subwavelength FP-like resonance in the slits also red shifts significantly. In addition, Fig. 4(a) suggests that the electromagnetic pressure is very sensitive to the spacer thickness: the smaller the thickness, the larger the electromagnetic pressure. This is mainly due to the fact that the energy density of electromagnetic fields in the spacer will increase as the spacer thickness decreases. Thus, according to Eq. (9), the electromagnetic force will be enhanced. We expect that the electromagnetic field will induce an oscillating force in the system. Suppose that the metallic slit grating is fixed and the planar metal film is connected to a spring or a soft medium, then the net attractive force caused by the incident light can drive the planar metal film. The motion of planar film will change the spacer thickness, and then the resonance wavelength will be shifted. Consequently, the reflection of light and the electromagnetic force are modulated dynamically. This feature may be utilized to drive the mechanical oscillations or excite the elastic waves. The mechanical oscillation and elastic waves can also be excited efficiently by a pulsed light beam or incident light with a modulated intensity. 

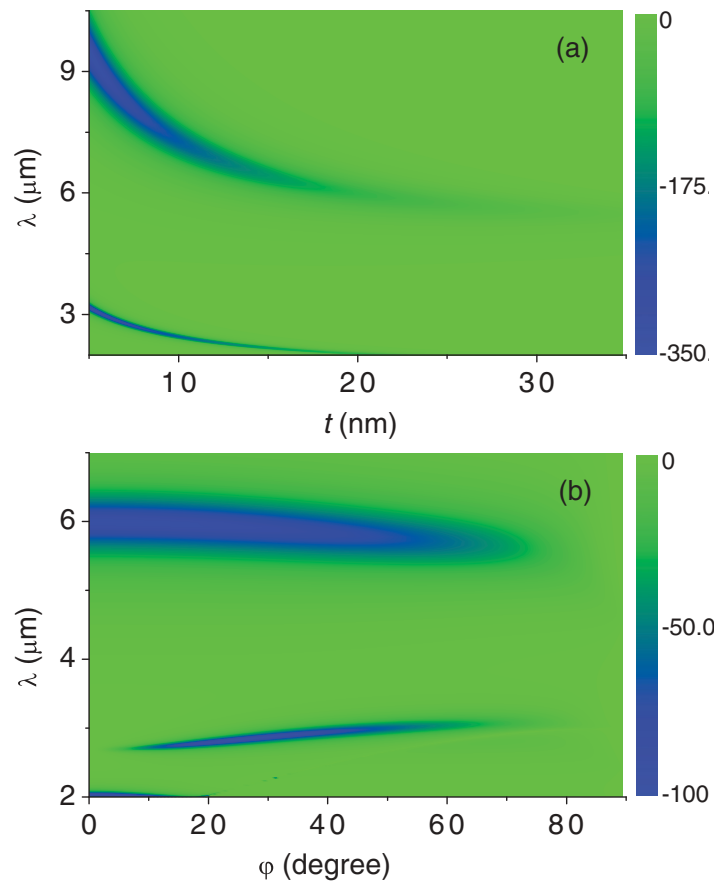

FIG. 4. (Color online) Normalized electromagnetic pressure in the infrared regime as a function of wavelength and (a) spacer thickness $t(\varphi=0)$ or (b) incident angle $\varphi(t=20 \mathrm{~nm})$. Here, $d=1500 \mathrm{~nm}, a=50 \mathrm{~nm}$, and $h=80 \mathrm{~nm}$.

The normalized electromagnetic pressure as a function of wavelength and incident angle has also been calculated analytically (where $t=20 \mathrm{~nm}$ ), as shown in Fig. 4(b). With the increase of incident angle $\varphi$, the deep subwavelength FPlike resonance around $6000 \mathrm{~nm}$ blueshifts slightly; however, the electromagnetic pressure reduces obviously, especially for the larger incident angle (note that the incident photon momentum in the surface-normal direction also reduces with $\varphi)$. By calculation, we found that the FP-like resonance factor $f_{p}$ and the electromagnetic fields in the slits and spacer decrease with the incident angle, which further reduces the electromagnetic pressure. Additionally, with the increase of $\varphi$, another deep subwavelength FP-like resonance near $3000 \mathrm{~nm}$ also appears in the slits. The presence of this resonance mode is correlated with the second-order FP resonance in the spacer (which is inactive at normal incidence). ${ }^{16}$ The FP-like resonance factor and field amplitudes were found to increase first with $\varphi$, but it then degenerates for the larger incident angle. This agrees with the variation character of electromagnetic pressure around $3000 \mathrm{~nm}$. These results provide the opportunity for manipulating the electromagnetic pressure with the incident angle.

\section{B. Electromagnetic pressure in the microwave regime}

In the microwave band the penetration depth of electromagnetic fields into the metal becomes large, and the role of electron kinetic energy is reduced. ${ }^{30}$ Thus the electromagnetic pressure here will be different from that observed in the infrared regime. Here, without loss of generality, the structure parameters are fixed as $d=20 \mathrm{~mm}$ and $a=1 \mathrm{~mm}$. The relative permittivity of the incident side, the slit inside, and the spacer
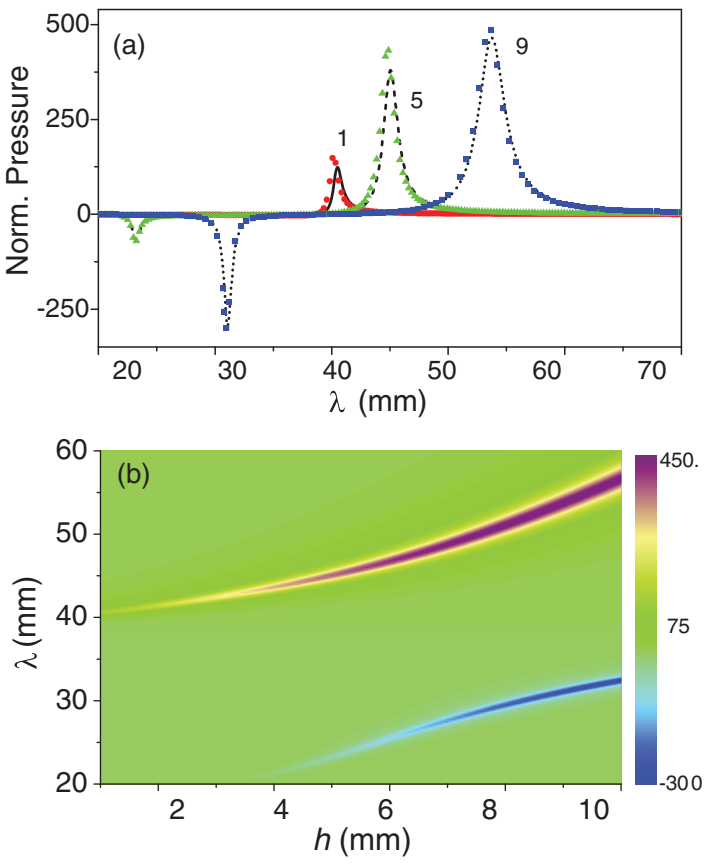

FIG. 5. (Color online) (a) Normalized electromagnetic pressure in the microwave band for different slit lengths $(h=1,5$, and $9 \mathrm{~mm}$ ). The lines and symbols represent the analytical and numerical results, respectively. (b) Normalized electromagnetic pressure in the microwave band as a function of wavelength and slit length. Here, the structure parameters are fixed as $d=20 \mathrm{~mm}, a=1 \mathrm{~mm}$, and $t=0.1 \mathrm{~mm}$.

region were still set as unity; the permittivity of gold was modeled as $\varepsilon_{m}=1+i \sigma / \varepsilon_{0} \omega$, where $\sigma=4.1 \times 10^{7} \mathrm{~S} / \mathrm{m}$ is the conductivity of a real metal. ${ }^{31}$

Figure 5(a) presents the analytically calculated (lines) and numerically simulated (symbols) normalized electromagnetic pressure for different slit lengths $h=1,5$, and $9 \mathrm{~mm}$ (here the spacer thickness is set as $t=0.1 \mathrm{~mm}$ ). Again, a good agreement between them can be obtained. Figure 5(a) suggests that, for the slit length $h=1 \mathrm{~mm}$, a positive peak of electromagnetic pressure (with a normalized value of $\sim 125$ ) appears around the wavelength $41 \mathrm{~mm}$. With the increase of $h$, this peak red shifts with the magnitude growing significantly. This is just opposite to the result in the infrared regime, where the electromagnetic pressure reduces with $h$ (see Fig. 2). For the slit length $h=9 \mathrm{~mm}$, the electromagnetic pressure reaches $\sim 465$ at the wavelength $54 \mathrm{~mm}$, which is one order of magnitude larger than that obtained with a single patch cavity. ${ }^{14}$ More interestingly, with the variation of slit length, a negative electromagnetic pressure also appears, which red shifts and grows in magnitude as $h$ increases. For $h=9 \mathrm{~mm}$, a negative pressure up to -300 can be achieved at the wavelength $31 \mathrm{~mm}$. Such strong negative pressure in the microwave band has not been found previously. For clarity, the normalized electromagnetic pressure as a function of wavelength and slit thickness is shown in Fig. 5(b), where the (upper) purple- and (lower) blue-colored part highlights the positive and negative pressure, respectively. We also would like to point out that, here, the sign of electromagnetic pressure may change at a specific wavelength, which depends on the details such as the geometrical parameters. For example, for $h=9 \mathrm{~mm}$, the 

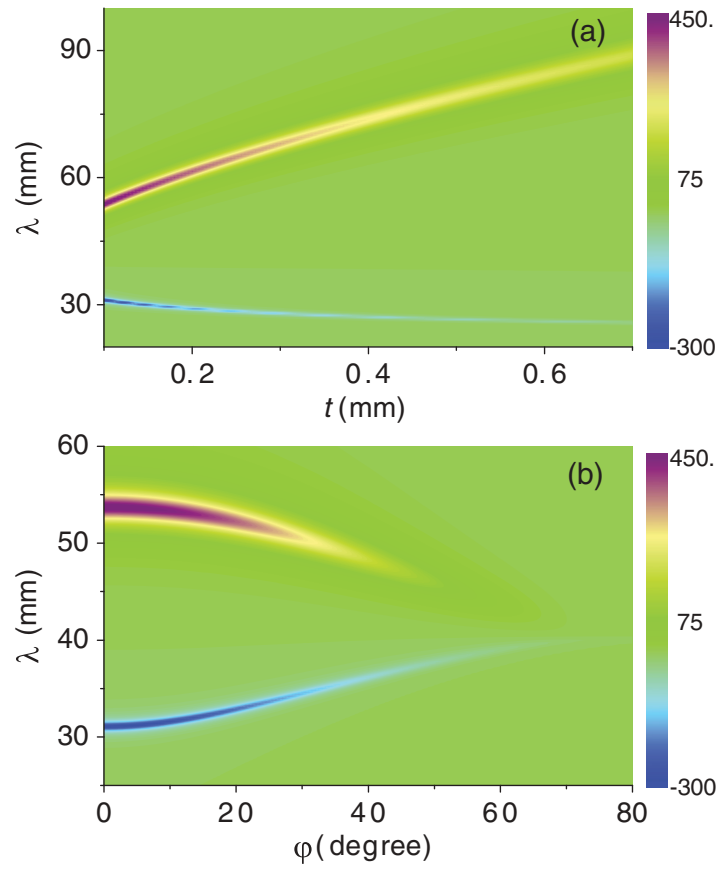

FIG. 6. (Color online) Normalized electromagnetic pressure in the microwave band as a function of wavelength and (a) spacer thickness $t(\varphi=0)$ or (b) incident angle $\varphi(t=0.1 \mathrm{~mm})$. Here, $d=20 \mathrm{~mm}, a=1 \mathrm{~mm}$, and $h=9 \mathrm{~mm}$ (the thickness of planar film was set as $3 \mathrm{~mm}$ in the simulation).

electromagnetic pressure is nearly zero and changes the sign at around the wavelength of $40 \mathrm{~mm}$. At that point, the system is off resonance, and the electromagnetic fields only weakly penetrate into the air spacer. Moreover, the averaged electric energy density in the spacer is balanced by the magnetic counterpart, thus giving rise to a null electromagnetic pressure.

In the microwave regime, as the role of the kinetic energy of oscillating free electrons is not significant, the electromagnetic pressure is usually positive (in a single metal patch cavity, a weak leakage of electric field around the cavity edge suppresses the attractive force). ${ }^{14}$ But here, the subwavelength slits, which can store electromagnetic energy, may give rise to a significant modulation of electromagnetic pressure. At the longer wavelength $(\lambda>40 \mathrm{~mm})$, the positive peak of pressure is due to the zero-order deep subwavelength FP-like resonance of the slits. In this case the current on the slit walls is very weak, and a lot of electric field energy is confined in the slits [similar to Figs. 3(a) and 3(b)]. Thus, the electromagnetic pressure is positive and increases with the slit length because more and more electric energy will be trapped in the slits as $h$ gets larger. Nonetheless, at the shorter wavelength, the enhanced pressure is due to the first-order FP-like resonance of the slits [which is located between the zero-phase point A and point B; refer to the inset of Fig. 2(a)]. In this case strong antiparallel currents are also generated on the slit walls, thus producing the magnetic energy in the slits [which also increases with the slit length $h$; similar to Figs. 3(c) and 3(d)]. This accounts for the appearance and increase of the strong negative force in the microwave regime.

The normalized electromagnetic pressure in the microwave band as a function of wavelength and spacer thickness is calculated and shown in Fig. 6(a) (the slit length is set as $h=9 \mathrm{~mm}$ ). Different from the infrared regime [see Fig. 4(a)], here an increase of spacer thickness $t$ leads to a significant red shift of the longer FP-like resonance. ${ }^{16}$ The electromagnetic pressure is found to decrease with $t$, in accordance with that of the infrared regime. In addition, Fig. 6(b) presents the calculated normalized electromagnetic pressure as a function of wavelength and incident angle (where $t=0.1 \mathrm{~mm}$ ). With the increase of incident angle, the zeroorder and first-order subwavelength FP-like resonance shifts to the shorter and longer wavelength, respectively. Moreover, the electromagnetic pressure also decreases with the incident angle significantly. This angle dependence may be useful for the manipulation of electromagnetic pressure in the microwave band.

\section{CONCLUSION}

In summary the electromagnetic pressure in a sandwiched reflection grating has been investigated. The plasmonic structure can support deep subwavelength FP-like resonances and hence induce a strong electromagnetic pressure. A theoretical formula has been derived and used to study the effect in different frequency bands. We found that the deep-subwavelength FP cavities can provide a new degree of freedom for manipulating the electromagnetic force. In the infrared regime the pressure is negative and controllable with the FP cavity length. In the microwave band, in addition to a commonly observed positive pressure, a strong negative electromagnetic pressure was also demonstrated.

\section{ACKNOWLEDGMENTS}

This work was supported by the Hong Kong Research Grants Council (Grant No. 600209), the National Basic Research Program of China (Grant No. 2012CB921502), the HKUST internal Grant No. SRFI11SC07, and the National Natural Science Foundation of China (Grant No. 11174146). Computation resources were provided by the Hong Kong Shun Hing Education and Charity Fund.

\footnotetext{
*cphuang@njut.edu.cn

†phchan@ust.hk

${ }^{1}$ A. Ashkin, Optical Trapping and Manipulation of Neutral Particles

Using Lasers (World Scientific Publishing, New Jersey, 2006).

${ }^{2}$ A. Ashkin, Phys. Rev. Lett. 24, 156 (1970).
}

${ }^{3}$ J. Chen, J. Ng, Z. Lin, and C. T. Chan, Nat. Photon. 5, 531 (2011).

${ }^{4}$ T. J. Kippenberg, H. Rokhsari, T. Carmon, A. Scherer, and K. J. Vahala, Phys. Rev. Lett. 95, 033901 (2005).

${ }^{5}$ A. Schliesser, P. DelHaye, N. Nooshi, K. Vahala, and T. Kippenberg, Phys. Rev. Lett. 97, 243905 (2006). 
${ }^{6}$ M. Eichenfield, C. P. Michael, R. Perahia, and O. Painter, Nat. Photon. 1, 416 (2007).

${ }^{7}$ T. J. Kippenberg and K. J. Vahala, Science 321, 1172 (2008).

${ }^{8}$ M. Eichenfield, R. Camacho, J. Chan, K. J. Vahala, and O. Painter, Nature 459, 550 (2009).

${ }^{9}$ D. Van Thourhout and J. Roels, Nat. Photon. 4, 211 (2010).

${ }^{10}$ R. Sainidou and F. J. Garcia de Abajo, Phys. Rev. Lett. 101, 136802 (2008).

${ }^{11}$ D. Woolf, M. Loncar, and F. Capasso, Opt. Express 17, 19996 (2009).

${ }^{12}$ X. Yang, Y. Liu, R. F. Oulton, X. Yin, and X. Zhang, Nano Lett. 11, 321 (2011).

${ }^{13}$ H. Liu, J. Ng, S. B. Wang, Z. F. Lin, Z. H. Hang, C. T. Chan, and S. N. Zhu, Phys. Rev. Lett. 106, 087401 (2011).

${ }^{14}$ S. B. Wang, J. Ng, H. Liu, H. H. Zheng, Z. H. Hang, and C. T. Chan, Phys. Rev. B 84, 075114 (2011).

${ }^{15}$ J. Zhang, K. F. MacDonald, and N. I. Zheludev, Phys. Rev. B 85, 205123 (2012).

${ }^{16}$ C. P. Huang, X. G. Yin, Y. Zhang, S. B. Wang, Y. Y. Zhu, H. Liu, and C. T. Chan, Phys. Rev. B 85, 235410 (2012).

${ }^{17}$ A. P. Hibbins, J. R. Sambles, C. R. Lawrence, and J. R. Brown, Phys. Rev. Lett. 92, 143904 (2004).

${ }^{18}$ Y. Todorov, A. M. Andrews, I. Sagnes, R. Colombelli, P. Klang, G. Strasser, and C. Sirtori, Phys. Rev. Lett. 102, 186402 (2009).

${ }^{19}$ Z. Y. Wei, H. Q. Li, Y. Cao, C. Wu, J. Z. Ren, Z. H. Hang, H. Chen, D. Z. Zhang, and C. T. Chan, New J. Phys. 12, 093020 (2010).

${ }^{20}$ P. Jouy, Y. Todorov, A. Vasanelli, R. Colombelli, I. Sagnes, and C. Sirtori, Appl. Phys. Lett. 98, 021105 (2011).

${ }^{21}$ For the secondary slit mode, the field decaying efficiency in the slits can be expressed as $e^{i q h}=e^{i \sqrt{k_{0}^{2} \varepsilon_{s}-(\pi / a)^{2}} h} \approx e^{-\pi h / a}$. For the slit with $h \geqslant a$, less than $0.2 \%$ of the secondary mode energy can tunnel through the slit, which will make very little contribution to the electromagnetic pressure.

${ }^{22}$ H. Lochbihler, Phys. Rev. B 50, 4795 (1994).

${ }^{23}$ S. Collin, F. Pardo, and J. Pelouard, Opt. Express 15, 4310 (2007).

${ }^{24}$ J. R. Suckling, A. P. Hibbins, M. J. Lockyear, T. W. Preist, and J. R. Sambles, Phys. Rev. Lett. 92, 147401 (2004).

${ }^{25}$ A. Wirgin and A. A. Maradudin, Prog. Surf. Sci. 22, 1 (1986).

${ }^{26}$ A. Barbara, P. Quemerais, E. Bustarret, and T. Lopez-Rios, Phys. Rev. B 66, 161403(R) (2002).

${ }^{27}$ As mentioned previously for the evanescent order, $\left|\rho_{m}\right|$ can be larger than unity. Thus the poles of $\mu_{m}$, corresponding to $1-\rho_{m}^{2} e^{2 i k_{0} v_{m} t}=$ 0 , may be reached. This gives the extended SPP resonance condition $k_{0} \sqrt{\varepsilon_{1}} \sin \varphi+2 \pi m / d= \pm k_{0} n_{t}$, where $n_{t}$ is the effective index of the region beneath the metal stripes.

${ }^{28}$ J. D. Jackson, Classical Electrodynamics (Wiley, New York, 1962).

${ }^{29}$ The collision frequency used here is slightly larger than the infrared value $\gamma=4.084 \times 10^{13} \mathrm{rad} / \mathrm{s}$; see M. A. Ordal, R. J. Bell, R. W. Alexander, Jr., L. L. Long, and M. R. Querry, Appl. Opt. 24, 4493 (1985).

${ }^{30}$ The ratio between the kinetic energy of free electrons and magnetic energy is $\eta_{e m}=L_{e} / L_{m}$, where $L_{e}$ and $L_{m}$ are the kinetic inductance and Faraday inductance, respectively. For a metal with the thickness larger than the skin depth $\delta, L_{e}$ and $\eta_{e m}$ will be inversely proportional to $\delta$. From the visible to mid-infrared band, $\delta$ is almost a constant, and thus the kinetic inductance plays an equal role. However, at the long wavelength such as the microwave band, $\delta$ is large and increases with the wavelength, thus the kinetic energy becomes negligible compared with the magnetic energy.

${ }^{31}$ D. M. Pozar, Microwave Engineering, 3rd ed. (Wiley, Hoboken, New Jersey, 1998). 